\title{
Hall Current And Dufour Effects On Mhd Flow Of A Micropolar Fluid Past A Vertical Plate In The Presence Of Radiation Absorption And Chemical Reaction
}

\author{
K. Surya Narayana Reddy ${ }^{1}$, M. Sreedhar Babu ${ }^{2}$, S. Vijaya Kumar Varma ${ }^{3}$ \\ and N. Bhaskar Reddy ${ }^{4}$ \\ ${ }^{1,3,4}$ Department of Mathematics, Srivenkateswara University, Tirupati-517502, Chittoor District, A. P., India \\ ${ }^{2}$ Department of Mathematics, Yogi Vemana University, Kadapa-516001, kadapa District, A. P., India
}

\begin{abstract}
Heat and mass transfer effects on an unsteady MHD flow of a chemically reacting micropolar fluid over an infinite vertical porous plate through a porous medium with Hall effects and thermal radiation in the presence of radiation absorption and heat sink are studied. The governing system of partial differential equations is transformed to dimensionless equations using dimensionless variables. The dimensionless equations are then solved analytically using the perturbation technique to obtain the expressions for velocity, microrotation, temperature and concentration. With the help of graphs, the effects of the various important parameters entering into the problem on the velocity, microrotation, temperature and concentration fields within the boundary layer are discussed. Also the effects of the pertinent parameters on the skin friction coefficient and rates of heat and mass transfer in terms of the Nusselt number and Sherwood number are presented numerically in a tabular form.
\end{abstract}

Keywords: Micropolar fluid, Perturbation technique, Heat and mass transfer, Hall effect, Porous medium, radiation absorption and heat sink.

\section{Introduction}

Micropolar fluids are fluids of microstructure. They represent fluids consisting of rigid, randomly oriented, or spherical particles suspended in a viscous medium, where deformation of fluids particles is ignored. The dynamics of micropolar fluids, originated from the theory of Eringen [1-3], has been a popular area of research due to its application in a number of processes that occur in industry. Such applications include polymeric fluids, real fluids with suspensions, liquid crystal, animal blood, and exotic lubricants. Extensive reviews of theory ofmicropolar fluids and its applications can be found in review articles by Ariman et al. $[4,5]$ and recent books by Lukaszewicz [6] and Eringen [7].

The current development of magnetohydrodynamics application is toward a strong magnetic field (so that the influence of electromagnetic force is noticeable) and toward a low density of the gas (such as in space flight and in nuclear fusion research). Under this condition, the Hall current becomes important. The rotating flow of an electrically conducting fluid in the presence of a magnetic field is encountered in geophysical, cosmical fluid dynamics, medicine and biology. Application in biomedical engineering includes cardiac MRI, ECG, etc. Mohamed et al [8] studied the thermal instability in a rotating micropolar viscoelastic fluid layer under the effect of electric field. Transient MHD free convection from an infinite vertical porous plate in a rotating system with mass transfer and Hall current was investigated by Ahmed and Kalita [9]. Hayat et al.[10] studied the Rotating flow of a third grade fluid in a porous space with Hall current. Neela Rani and Tomar [11] analyzes the thermal convection problem of micropolar fluid subjected to hall current.

The energy flux caused by composition gradient is called the Dufour or diffusion-thermal effect. The diffusion-thermo (Dufour) effect was found to be of considerable magnitude such that it cannot be ignored, Eckert and Drake [12]. In view of the importance of this diffusion-thermo effect, Alam and Rahmam [13] studied the Dufour and Soret effects on study MHD free convective heat and mass transfer flow past a semiinfinite vertical porous plate embedded in a porous medium. Sedeek [14] has considered thermal-diffusion and diffusion-thermo effects on mixed free-forced convective flow and mass transfer over accelerating surface with a heat source in the presence of suction and blowing in the case of variable viscosity. Dufour and Soret effects on steady MHD free convection and mass transfer fluid flow through a porous medium in a rotating system were studied recently by Nazmul and Mahmud [15]. Kafoussias and Williams [16] presented thermal-diffusion and diffusion-thermo effects on mixed free forced convective and mass transfer boundary layer flow with temperature dependent.

The heat source/sink effects in thermal convection, are significant where there may exist a high temperature differences between the surface (e.g. space craft body) and the ambient fluid. Heat generation is also important in the context of exothermic or endothermic chemical reactions. Singh et al. [17], investigated the 
effect of volumetric heat generation/absorption on mixed convection stagnation point flow on an isothermal vertical plate in porous media. Das et al. [18] investigated the hydromagnetic convective flow past a vertical porous plate through a porous medium with suction and heat source. Das et al. [19] have discussed the Mass transfer effects on unsteady hydro magnetic convective flow past a vertical porous plate in a porous medium with heat source. Khan [20] studied the effect of heat transfer on a viscoelastic fluid flow over a stretching sheet with heat source/sink, suction/blowing and radiation. Pal and Talukdar [21] studied the unsteady MHD heat and mass transfer along with heat source past a vertical permeable plate using a perturbation analysis, where the unsteadiness is caused by the time dependent surface temperature and concentration.

The porous media heat and mass transfer problems have several practical engineering applications such as geothermal systems, crude oil extraction, and ground-water pollution. The study of chemical reaction heat transfer in porous medium has important applications such as in tabular reactors, oxidation of solid materials, and synthesis of ceramic materials. Elgazery [22] numerically analyzed the effects of chemical reaction, Hall, ion-slip currents, variable viscosity and variable thermal diffusivity on the problem of magnetomicropolar fluid flow, heat and mass transfer with suction and blowing through a porous medium. Oahimire and Olajuwon [23] studied the effect of Hall current and thermal radiation on heat and mass transfer of a chemically reacting MHD flow of a micropolar fluid through a porous medium. Satya Narayana et al. [24] investigated the effects of Hall current and radiation absorption on MHD micropolar fluid in a rotating system. Motsa and Shateyi [25] analyzes the effects of chemical reaction, hall, and ion-slip currents on MHD micropolar fluid flow with thermal diffusivity using a novel numerical technique. Salem [26] studied the effects of variable viscosity, viscous dissipation and chemical reaction on heat and mass transfer flow of MHD micropolar fluid along a permeable stretching sheet in a Non-Darcian porous medium.

The object of the present chapter is to analyze the heat and mass transfer effects on an unsteady MHD flow of a chemically reacting micropolar fluid over an infinite vertical porous plate through a porous medium with Hall effects and thermal radiation in the presence of radiation absorption and heat sink. The governing equations are solved using perturbation technique. The effects of various governing parameters on the fluid velocity, temperature, concentration, skin-friction coefficient, Nusselt number and Sherwood number are shown in figures and tables and further analyzed in detail.

\section{Formulation Of The Problem}

We consider an unsteady flow of an incompressible, electrically conducting micropolar fluid over an infinite vertical porous plate through a porous medium, subjected to a constant transverse magnetic field $\mathrm{B}_{0}$ in the presence of thermal and concentration buoyancy effects.

The induced magnetic field is assumed to be negligible compared to the applied magnetic field. The $x^{\prime}$ -axis is taken along the planar surface in the upward direction and the $y^{\prime}$-axis is taken to be normal to it. Due to the infinite plane surface assumption, the flow variables are functions of $y^{\prime}$ and the $t^{\prime}$ only. The plate is subjected to a constant suction velocity $\mathrm{V}_{0}$.

According to the usual Boussinesq and boundary-layer approximation, the governing equations for this problem can be written as follows:

$$
\begin{aligned}
& \frac{\partial v^{\prime}}{\partial t^{\prime}}=0 \\
& \frac{\partial u^{\prime}}{\partial t^{\prime}}+v^{\prime} \frac{\partial u^{\prime}}{\partial y^{\prime}}=\left(v+v_{r}\right) \frac{\partial^{2} u^{\prime}}{\partial y^{\prime 2}}+v_{r} \frac{\partial N_{1}^{\prime}}{\partial y^{\prime}} \\
& -\frac{\sigma \beta_{0}^{2}\left(u^{\prime}+m w^{\prime}\right)}{\rho\left(1+m^{2}\right)}-\frac{v}{K^{\prime}} u^{\prime}+g \beta_{T}\left(T^{\prime}-T_{\infty}^{\prime}\right)+g \beta_{C}\left(C^{\prime}-C_{\infty}^{\prime}\right) \\
& \frac{\partial w^{\prime}}{\partial t^{\prime}}+v^{\prime} \frac{\partial w^{\prime}}{\partial y^{\prime}}=\left(v+v_{r}\right) \frac{\partial^{2} w^{\prime}}{\partial y^{\prime 2}}-v_{r} \frac{\partial N_{2}{ }^{\prime}}{\partial y^{\prime}}-\frac{\sigma \beta_{0}^{2}\left(w^{\prime}-m u^{\prime}\right)}{\rho\left(1+m^{2}\right)}-\frac{v}{K^{\prime}} w^{\prime} \\
& \rho j^{\prime}\left(\frac{\partial N_{1}^{\prime}}{\partial t^{\prime}}+v^{\prime} \frac{\partial N_{1}{ }^{\prime}}{\partial y^{\prime}}\right)=\gamma \frac{\partial^{2} N_{1}^{\prime}}{\partial y^{\prime 2}} \\
& \rho j^{\prime}\left(\frac{\partial N_{2}^{\prime}}{\partial t^{\prime}}+v^{\prime} \frac{\partial N_{2}{ }^{\prime}}{\partial y^{\prime}}\right)=\gamma \frac{\partial^{2} N_{2}{ }^{\prime}}{\partial y^{\prime 2}}
\end{aligned}
$$




$$
\begin{aligned}
& \frac{\partial T^{\prime}}{\partial t^{\prime}}+v^{\prime} \frac{\partial T^{\prime}}{\partial y^{\prime}}=\frac{k}{\rho c_{p}} \frac{\partial^{2} T^{\prime}}{\partial y^{\prime 2}}-\frac{1}{\rho c_{p}} \frac{\partial q_{r}}{\partial y^{\prime}}+\frac{D_{m} k_{T}}{c_{s} c_{p}} \frac{\partial^{2} C^{\prime}}{\partial y^{\prime 2}}-\frac{Q^{\prime}}{\rho c_{p}}\left(T^{\prime}-T_{\infty}^{\prime}\right)+\frac{Q_{1}^{\prime}}{\rho c_{p}}\left(C^{\prime}-C_{\infty}^{\prime}\right) \\
& \frac{\partial C^{\prime}}{\partial t^{\prime}}+v^{\prime} \frac{\partial C^{\prime}}{\partial y^{\prime}}=D \frac{\partial^{2} C^{\prime}}{\partial y^{\prime 2}}-K r^{\prime}\left(C^{\prime}-C_{\infty}^{\prime}\right)
\end{aligned}
$$

The initial and boundary conditions are:

$$
\begin{aligned}
& u^{\prime}=L^{\prime}\left(\frac{\partial u^{\prime}}{\partial y^{\prime}}\right), v^{\prime}=0, w^{\prime}=L^{\prime}\left(\frac{\partial u^{\prime}}{\partial y^{\prime}}\right), N_{1}^{\prime}=-n \frac{\partial u^{\prime}}{\partial y^{\prime}}, N_{2}^{\prime}=-n \frac{\partial w^{\prime}}{\partial y^{\prime}} \\
& T^{\prime}=T_{\infty}^{\prime}+\left(T^{\prime}{ }_{w}-T_{\infty}^{\prime}\right) e^{i \omega^{\prime} t^{\prime}}, C^{\prime}=C_{\infty}^{\prime}+\left(C^{\prime}{ }_{w}-C^{\prime}{ }_{\infty}\right) e^{i \omega^{\prime} t^{\prime}}, \text { at } y^{\prime}=0 \\
& u^{\prime} \rightarrow 0, v^{\prime} \rightarrow 0, w^{\prime} \rightarrow 0, N^{\prime}{ }_{1} \rightarrow 0, N_{2}^{\prime} \rightarrow 0, T^{\prime} \rightarrow T_{\infty}^{\prime}, C^{\prime} \rightarrow C_{\infty}^{\prime}, \text { as } y^{\prime} \rightarrow \infty
\end{aligned}
$$

where $u^{\prime}, v^{\prime}$ and $w^{\prime}$ are velocity components along $x^{\prime}, y^{\prime}$ and $z^{\prime}$-axis respectively, $N_{1}{ }^{\prime}$ and $N_{2}{ }^{\prime}$ are microrotation components along $x^{\prime}$ and $z^{\prime}$ axis respectively, $v$ is the kinematic viscosity, $v_{r}$ is the kinematic micro-rotation viscosity, $q_{r}$ is the radiative heat flux, $\mathrm{g}$ is the acceleration due to gravity, $\beta_{T}$ and $\beta_{C}$ are the coefficients of thermal expansion and concentration expansion respectively, $T^{\prime}$ is the dimensional temperature of the fluid, $T^{\prime}{ }_{w}$ and $T^{\prime}{ }_{\infty}$ denote the temperature at the plate and temperature far away from the plate respectively, $C^{\prime}$ is the dimensional concentration of the solute, $C^{\prime}{ }_{w}$ and $C^{\prime}{ }_{\infty}$ are concentration of the solute at the plate and concentration of the solute far from the plate respectively, $K^{\prime}$ is the permeability of the porous medium, $c_{p}$ is the specific heat at constant pressure at $\mathrm{p}, \mathrm{k}$ is the thermal conductivity of the medium, $\rho$ is the density of the fluid, $j^{\prime}$ is the micro-inertia density or micro-inertia per unit mass, $\gamma$ is the spin gradient viscosity, $L^{\prime}$ is the characteristic length, $\omega^{\prime}$ is the dimensional frequency of oscillation, $\sigma$ is the electrical conductivity, $\mathrm{m}$ is the Hall current parameter, $Q^{\prime}$ is additional heat source constant, $Q_{1}{ }^{\prime}$ is the radiation absorption rate constant, $D_{m}$ is the molecular diffusivity, $k_{T}$ is the thermo diffusion ratio, $c_{s}$ is the concentration susceptibility, $K r^{\prime}$ is the rate of chemical reaction and $\mathrm{D}$ is the molecular diffusivity.

The constant that is related to microgyration vector and shear stress is $\mathrm{n}$. Further, $0 \leq n \leq 1$. The case $\mathrm{n}=0$ represents concentrated particle flows in which the microelement close to the wall surface is unable to rotate. This case is also known as the strong concentration of microelements. The case $\mathrm{n}=0.5$ indicates the vanishing of anti-symmetric part of the stress tensor and denotes weak concentration of microelements. The case $\mathrm{n}=1$ is used for the modelling of turbulent boundary layer flows. We shall consider $\mathrm{n}=0$ and $\mathrm{n}=0.5$.

Following Rosseland approximation, the radiative heat flux $q_{r}$ is modeled as

$$
q_{r}=\frac{4 \sigma^{\prime}}{3 k^{\prime}} \frac{\partial T^{\prime 4}}{\partial y^{\prime}}
$$

where $\sigma^{\prime}$ is the Stefan-Boltzman constant and $k^{\prime}$ is the mean absorption coefficient. Assuming that the difference in temperature within the flow is small such that $T^{\prime 4}$ can be expressed as a linear combination of the temperature, we expand $T^{, 4}$ in Taylor's series about $T_{\infty}^{\prime 4}$ as follows:

$$
T^{\prime 4}=T_{\infty}^{\prime 4}+4 T_{\infty}^{\prime 3}\left(T^{\prime}-T_{\infty}^{\prime}\right)+6 T_{\infty}^{\prime 2}\left(T^{\prime}-T_{\infty}^{\prime}\right)^{2}+---
$$

and neglecting higher order terms beyond the first degree in $\left(T^{\prime}-T_{\infty}^{\prime}\right)$, we have

$$
T^{\prime 4} \approx-3 T_{\infty}^{\prime 4}+4 T_{\infty}^{\prime 3} T^{\prime}
$$

Differentiating equation (2.10) with respect to $y^{\prime}$ and using equation (2.12) to obtain

$$
\frac{\partial q_{r}}{\partial y^{\prime}}=-\frac{16 T_{\infty}^{\prime} \sigma^{\prime}}{3 k^{\prime}} \frac{\partial^{2} T^{\prime}}{\partial y^{\prime 2}}
$$


Let us introduce the following dimensionless variables:

$$
\begin{aligned}
& u=\frac{u^{\prime}}{V_{0}}, v=\frac{v^{\prime}}{V_{0}}, w=\frac{w^{\prime}}{V_{0}}, \eta=\frac{V_{0} y^{\prime}}{v}, N_{1}=\frac{v N_{1}^{\prime}}{V_{0}^{2}}, N_{2}=\frac{v N_{2}^{\prime}}{V_{0}^{2}}, t=\frac{t^{\prime} V_{0}^{2}}{4 v} \\
& \omega=\frac{4 v \omega^{\prime}}{V_{0}^{2}}, h=\frac{V_{0} L^{\prime}}{v}, \theta=\frac{T^{\prime}-T_{\infty}^{\prime}}{T^{\prime}{ }_{w}-T_{\infty}^{\prime}}, C=\frac{C^{\prime}-C_{\infty}^{\prime}}{C^{\prime}{ }_{w}-C_{\infty}^{\prime}}, j=\frac{V_{0}^{2} j^{\prime}}{v^{2}}, \beta=\frac{v_{r}}{v} \\
& N r=\frac{16 T_{\infty}^{\prime} \sigma^{\prime}}{3 k k^{\prime}}, M=\frac{\sigma B_{0}^{2} v}{\rho V_{0}^{2}}, G r=\frac{v \beta_{T} g\left(T^{\prime}{ }_{w}-T_{\infty}^{\prime}\right)}{V_{0}^{3}}, G c=\frac{v \beta_{C} g\left(C^{\prime}{ }_{w}-C_{\infty}^{\prime}\right)}{V_{0}^{3}} \\
& \operatorname{Pr}=\frac{v \rho c_{p}}{k}, S c=\frac{v}{D}, K=\frac{K^{\prime} V_{0}^{2}}{v^{2}}, L=\frac{\gamma V_{0}^{2}}{\rho v^{2} j}, Q^{\prime}=\frac{Q v^{2}}{V_{0}^{2} k}, Q_{1}=\frac{Q_{1}^{\prime}\left(C^{\prime}{ }_{w}-C_{\infty}^{\prime}\right)}{\left(T^{\prime}{ }_{w}-T_{\infty}^{\prime}\right) V_{0}^{2}} \\
& n^{\prime}=\frac{n v}{V_{0}^{2}}, D u=\frac{D_{m} k_{T}\left(C^{\prime}{ }_{w}-C_{\infty}^{\prime}\right)}{c_{s} c_{p}\left(T^{\prime}{ }_{w}-T_{\infty}^{\prime}\right)}, K r=\frac{K r^{\prime} v}{V_{0}^{2}}
\end{aligned}
$$

where Gr is thermal Grashof number, Gc is the solutal Grashof number, $\mathrm{M}$ is the magnetic parameter, $\beta$ is dimensionless viscosity ratio, $\mathrm{Q}$ is the heat source parameter, $Q_{1}$ is the radiation absorption parameter, $\mathrm{Nr}$ is the thermal radiation parameter, $\mathrm{K}$ is the permeability parameter, $\mathrm{L}$ is the material parameter, Sc is the Schmidt number, Pr is the Prandtl number, Du is the Dufour number and $\mathrm{Kr}$ is the chemical reaction parameter.

Substituting equation (2.14) into equations (2.2)-(2.9) and using equations (2.1) and (2.13) yield the following dimensionless equations:

$$
\begin{aligned}
& \frac{1}{4} \frac{\partial u}{\partial t}-\frac{\partial u}{\partial \eta}=(1+\beta) \frac{\partial^{2} u}{\partial \eta^{2}}+\beta \frac{\partial N_{1}}{\partial \eta}-\frac{M}{1+m^{2}}(m w+u)+G r \theta+G c C-\frac{u}{K} \\
& \frac{1}{4} \frac{\partial w}{\partial t}-\frac{\partial w}{\partial \eta}=(1+\beta) \frac{\partial^{2} w}{\partial \eta^{2}}-\beta \frac{\partial N_{2}}{\partial \eta}-\frac{M}{1+m^{2}}(w-m u)-\frac{u}{K} \\
& \frac{1}{4} \frac{\partial N_{1}}{\partial t}-\frac{\partial N_{1}}{\partial \eta}=L \frac{\partial^{2} N_{1}}{\partial \eta^{2}} \\
& \frac{1}{4} \frac{\partial N_{2}}{\partial t}-\frac{\partial N_{2}}{\partial \eta}=L \frac{\partial^{2} N_{2}}{\partial \eta^{2}} \\
& \frac{1}{4} \frac{\partial \theta}{\partial t}-\frac{\partial \theta}{\partial \eta}=\frac{1}{\operatorname{Pr}}(1+N r) \frac{\partial^{2} \theta}{\partial \eta^{2}}+D u \frac{\partial^{2} C}{\partial \eta^{2}}-\frac{Q}{\operatorname{Pr}} \theta+Q_{1} C \\
& \frac{1}{4} \frac{\partial C}{\partial t}-\frac{\partial C}{\partial \eta}=\frac{1}{S c} \frac{\partial^{2} C}{\partial \eta^{2}}-K r C
\end{aligned}
$$

The corresponding boundary conditions are

$$
\begin{aligned}
& u=h \frac{\partial u}{\partial \eta}, v=0, w=h \frac{\partial w}{\partial \eta}, \theta=e^{i \omega t}, C=e^{i \omega t}, N_{1}=-n \frac{\partial u}{\partial \eta}, N_{2}=n \frac{\partial w}{\partial \eta} \text { at } \eta=0 \\
& u \rightarrow 0, v \rightarrow 0, w \rightarrow 0, \theta \rightarrow 0, C \rightarrow 0, N_{1} \rightarrow 0, N_{2} \rightarrow 0 \text { as } \eta \rightarrow \infty
\end{aligned}
$$

where $h=\frac{V_{0} L^{\prime}}{v}$ is the slip parameter.

By introducing $q=u+i w$ and $P=N_{1}+i N_{2}$ into equations 92.15) to (2.18) and simplifying we have

$$
\begin{aligned}
& \frac{1}{4} \frac{\partial q}{\partial t}-\frac{\partial q}{\partial \eta}=(1+\beta) \frac{\partial^{2} q}{\partial \eta^{2}}+i \beta \frac{\partial P}{\partial \eta}-\frac{M}{1+m^{2}}(1-i m) q+G r \theta+G c C-\frac{q}{K} \\
& \frac{1}{4} \frac{\partial P}{\partial t}-\frac{\partial P}{\partial \eta}=L \frac{\partial^{2} P}{\partial \eta^{2}} \\
& \frac{1}{4} \frac{\partial \theta}{\partial t}-\frac{\partial \theta}{\partial \eta}=\frac{1}{\operatorname{Pr}}(1+N r) \frac{\partial^{2} \theta}{\partial \eta^{2}}+D u \frac{\partial^{2} C}{\partial \eta^{2}}-\frac{Q}{\operatorname{Pr}} \theta+Q_{1} C
\end{aligned}
$$




$$
\frac{1}{4} \frac{\partial C}{\partial t}-\frac{\partial C}{\partial \eta}=\frac{1}{S c} \frac{\partial^{2} C}{\partial \eta^{2}}-K r C
$$

The corresponding boundary conditions are

$$
\begin{array}{lll}
q & =h \frac{\partial q}{\partial \eta}, \theta=e^{i \omega t}, C=e^{i \omega t}, P=i n \frac{\partial q}{\partial \eta} & \text { at } \quad \eta=0 \\
q \rightarrow 0, \theta \rightarrow 0, C \rightarrow 0, P \rightarrow 0 & \text { as } & \eta \rightarrow \infty
\end{array}
$$

\section{Method Of Solution}

In order to solve equations (2.23) to (2.26) subject to the boundary conditions (2.27) and (2.28), in the neighborhood of the plate, it is assumed that

$$
q=q_{0}(\eta) e^{i \omega t}, P=P_{0}(\eta) e^{i \omega t}, \theta=\theta_{0}(\eta) e^{i \omega t}, C=C_{0}(\eta) e^{i \omega t}
$$

Substituting Eq. (3.1) in to Eqs (2.23) to (2.26), we obtain the following set of equations

$$
\begin{aligned}
& a_{1} q_{0}{ }^{\prime \prime}+q_{0}{ }^{\prime}-a_{2} q_{0}=-G r \theta_{0}-G c C_{0}-i \beta P_{0}{ }^{\prime} \\
& L P_{0}{ }^{\prime}+P_{0}{ }^{\prime}-\frac{i \omega}{4} P_{0}=0 \\
& a_{3} \theta_{0}{ }^{\prime \prime}+\theta_{0}{ }^{\prime}-a_{4} \theta_{0}=-Q_{1} C_{0}-D u C_{0} " \\
& C_{0}{ }^{\prime}+S c C_{0}{ }^{\prime}-a_{5} C_{0}=0
\end{aligned}
$$

The corresponding boundary conditions are

$$
\begin{aligned}
& q_{0}=h \frac{\partial q_{0}}{\partial \eta}, \theta_{0}=1, C_{0}=1, P_{0}=i n \frac{\partial q_{0}}{\partial \eta} \text { at } \eta=0 \\
& q_{0}=0, \theta_{0}=0, C_{0}=0, P_{0}=0 \text { as } \eta \rightarrow \infty
\end{aligned}
$$

Solving equations (3.2) to (3.5) with the boundary conditions (3.6) and (3.7), we get the solutions as

Where

$$
\begin{aligned}
& q=\left(A_{1} e^{-m_{4} \eta}+A_{2} e^{-m_{1} \eta}+A_{3} e^{-m_{2} \eta}+A_{4} e^{-m_{3} \eta}\right) e^{i \omega t} \\
& P=B_{1} e^{i \omega t-m_{1} \eta} \\
& \theta=\left(a_{7} e^{-m_{2} \eta}+a_{8} e^{-m_{3} \eta}\right) e^{i \omega t} \\
& C=e^{i \omega t-m_{2} \eta}
\end{aligned}
$$

$$
\begin{aligned}
& m_{1}=\frac{1+\sqrt{1+i \omega L}}{2}, m_{2}=\frac{S c+\sqrt{S c^{2}+4 a_{5}}}{2}, m_{3}=\frac{1+\sqrt{1+4 a_{3} a_{4}}}{2 a_{3}} \\
& m_{4}=\frac{1+\sqrt{1+4 a_{1} a_{2}}}{2 a_{1}}, a_{1}=1+\beta, a_{2}=i\left(\frac{\omega}{4}-\frac{M m}{1+m^{2}}\right)+\frac{M}{1+m^{2}}+\frac{1}{K} \\
& a_{3}=\frac{1+N r}{\operatorname{Pr}}, a_{4}=\frac{i \omega}{4}+\frac{Q}{\operatorname{Pr}}, a_{5}=S c\left(\frac{i \omega}{4}+K r\right), a_{6}=-\left(Q_{1}+m_{2}^{2} D u\right) \\
& a_{7}=\frac{a_{6}}{a_{3} m_{2}^{2}-m_{2}-a_{4}}, a_{8}=1-a_{7}, A_{1}=-\frac{\left(A_{2}\left(1+h m_{1}\right)+A_{3}\left(1+h m_{2}\right)+A_{4}\left(1+h m_{3}\right)\right)}{1+h m_{4}} \\
& A_{2}=\frac{-m_{1} \beta n m_{4}\left(h m_{2} A_{4}+h m_{3} A_{3}+A_{3}+A_{4}\right)+\left(1+h m_{4}\right) \beta m_{1} n\left(m_{2} A_{4}+m_{3} A_{3}\right)}{\left(m_{1}^{2} a_{1}-m_{1}-a_{2}-\beta m_{1}^{2} n\right)\left(1+h m_{4}\right)+\beta m_{1} n m_{4}\left(1+h m_{1}\right)} \\
& A_{3}=-\frac{\left(G r a_{7}+G c\right)}{a_{1} m_{2}^{2}-m_{2}-a_{2}}, A_{4}=-\frac{G r a_{8}}{a_{1} m_{3}^{2}-m_{3}-a_{2}} \\
& B_{1}=-i n\left(A_{1} m_{4}+A_{2} m_{1}+A_{3} m_{2}+A_{4} m_{3}\right)
\end{aligned}
$$

The local skin friction coefficient, couple stress coefficient, Nusselt number and Sherwood number are important physical quantities of engineering interest.

The skin friction coefficient $C_{f}$ at the plate is given by

$$
C_{f}=\frac{\tau_{w}}{\rho V_{0}^{2}}=[1+(1-n) L] q^{\prime}(0)=-[1+(1-n) L]\left(A_{1} m_{4}+A_{2} m_{1}+A_{3} m_{2}+A_{4} m_{3}\right)
$$

where $\tau_{w}$ is the skin friction 
The couple stress coefficient $C^{\prime}{ }_{w}$ at the plate is written as

$$
C^{\prime}{ }_{w}=\frac{M_{w} v^{2}}{\gamma V_{0}^{3}}=P^{\prime}(0)=-m_{1} B_{1} e^{i \omega t}
$$

where $M_{w}$ is the plate couple stress.

The rate of heat transfer at the surface in terms of the Nusselt number is given by

$$
\begin{gathered}
N u=\frac{x\left(\frac{\partial T^{\prime}}{\partial y^{\prime}}\right)_{y^{\prime}=0}}{\left(T_{\infty}^{\prime}-T^{\prime}{ }_{w}\right)} \\
N u \operatorname{Re}^{-1}=-\theta^{\prime}(0)=\left(m_{2} a_{7}+m_{3} a_{8}\right) e^{i \omega t}
\end{gathered}
$$

The rate of mass transfer at the surface in terms of the local Sherwood number is given by

$$
\begin{gathered}
S h=\frac{x\left(\frac{\partial C^{\prime}}{\partial y^{\prime}}\right)_{y^{\prime}=0}}{\left(C_{\infty}^{\prime}-C^{\prime}{ }_{w}\right)} \\
S h \operatorname{Re}^{-1}=-(C)^{\prime}(0)=m_{1} e^{i \omega t}
\end{gathered}
$$

\section{Results And Discussion}

An unsteady three dimensional flow of an incompressible electrically conducting and chemically reacting micropolar fluid over an infinite vertical porous plate through a porous medium in the presence of radiation absorption and heat sink was studied. Numerical evaluation of the analytical solutions reported in the previous section was performed and the results are presented in graphical and tabular forms. This was done to illustrate the influence of various parameters involved. In this study, we have chosen $t=1$ and $\omega=0.01$ while other parameters are varied over a range.

The effect of magnetic field parameter on velocity distribution profiles across the boundary layer is presented in Fig. 1. It is obvious that the effect of increasing values of the magnetic field parameter (M) results in a decreasing velocity distribution across the boundary layer. This is due to the fact that the effect of a transverse magnetic field gives rise to a resistive type-force called the Lorentz force. The force has the tendency to slow the motion of the fluid. Fig. 2 displays the effect of Hall current parameter (m) on the translational velocity distribution profiles. It is noticed that the Hall current parameter increases the velocity. Figs. 3 and 4 illustrate the translational velocity profiles for different values of the Grashof number (Gr) and modified Grashof number $(\mathrm{Gc})$ respectively. It can be seen that an increase in $\mathrm{Gr}$ or $\mathrm{Gc}$ leads to a rise in velocity profiles. Fig. 5 depicts the effect of permeability of the porous medium parameter $(\mathrm{K})$ on translational velocity distribution profiles and it is obvious that as permeability parameter increases, the velocity increases along the boundary layer thickness which is expected since when the holes of porous medium become larger, the resistive of the medium may be neglected. Fig.6 illustrates the variation of slip parameter (h) with translational distribution profiles. It can be observed from the figure that the velocity increases with increasing value of the parameter near the plate and starts decreasing away from the plate. Fig. 7 shows the translational velocity profiles with different values of radiation parameter. And the effect of increasing the radiation parameter is to increase the translational velocity. This is because when the intensity of heat generated through thermal radiation is increased, the bond holding the components of the fluid particles is easily broken and the fluid velocity is increased. Fig. 8 displays the effect of heat sink parameter (Q) on the translational velocity distribution profiles. It is noticed that the heat sink parameter decreases the velocity. Fig. 9 depicts the effect of radiation absorption parameter $\left(\mathrm{Q}_{1}\right)$ on the translational velocity distribution profiles. It is noticed that the radiation absorption parameter increases the velocity. Fig. 10 shows the influence of the chemical reaction parameter $(\mathrm{Kr})$ on translational velocity profiles. The velocity decreases as the parameter increases.

Figs. 11(a) and (b) depict the microrotational velocity profiles for different values of magnetic field parameter respectively. The microrotational velocity distribution profiles decrease with an increase in the magnetic field parameter when $n=0.5$ while it remains constant when $n=0$ as shown in Fig. 11(b). It is clear from these figures that microrotational effect is more pronounced for $\mathrm{n}=0.5$ in comparison to when $\mathrm{n}=0$. Fig. 12 illustrates the microrotational velocity distribution for different values of Hall current parameter. The figure shows that as Hall current parameter increases, microrotational velocity increases. Figs. 13 and 14 elucidate that the effect of increasing Gr or Gc is to increase microrotational velocity. The effects due to permeability of the porous medium parameter on microrotational velocity are shown in Fig. 15. It is observed that as the parameter increases, the microrotational velocity increases. Fig. 16 shows that as the material parameter (L) increases, microrotational velocity decreases. Fig. 17 shows that the effect of increasing slip parameter is to decrease the 
microrotational velocity. Fig. 18 illustrates the effect of radiation parameter on microrotational velocity profiles. The profiles increase as the parameter increases. Fig. 19 shows that as the heat sink parameter increases, microrotational velocity decreases. Fig. 20 shows the influence of radiation absorption parameter on microrotational velocity profiles. It is observed that as radiation absorption parameter increases, the microrotational velocity increases. Fig. 21 illustrates the microrotational velocity distribution for different values of chemical reaction parameter. The figure shows that as chemical reaction parameter increases, the microrotational velocity decreases.

Fig.22 illustrates the effect of the thermal radiation parameter on the temperature. It is noticed that as the thermal radiation parameter increases, the temperature of the fluid increases. Fig.23 illustrates the effect of the heat sink parameter on the temperature. It is noticed that as the heat sink parameter increases, the temperature of the fluid decreases. Fig. 24 shows the variation of the thermal boundary-layer with the radiation absorption parameter. It is observed that the thermal boundary layer thickness increases with an increase in the radiation absorption parameter. Fig. 25 shows the variation of the thermal boundary-layer with the Dufour number $(\mathrm{Du})$. It is noticed that the thermal boundary layer thickness increases with an increase in the Dufour number. Figs. 26 and 27 show concentration distribution profiles for different values of Sc and $\mathrm{Kr}$. It can be noted from these figures that the concentration of the fluid decreases as the parameters increase.

Table 1 shows the effects of constant that is related to microgyration vector, magnetic field parameter, radiation parameter, Hall current parameter, heat sink parameter, radiation absorption parameter and chemical reaction parameter on skin friction coefficient and couple stress coefficient. It is observed that an increase in the value of $n$ decreases skin friction coefficient which is not surprising since $n=0$ represents strong concentration and $n=0.5$ represents weak concentration of the microelements while couple stress coefficient increases with an increase in $\mathrm{n}$. the skin friction increase while couple stress coefficient decrease with an increase in the parameter $\mathrm{M}$ and the opposite results were found when $\mathrm{m}$. An increase in radiation parameter and chemical reaction parameter increases both skin friction coefficient and couple stress coefficient while an increase in heat sink parameter and radiation absorption parameter decreases them. Table 2 shows the effect of the radiation parameter, heat sink parameter, radiation absorption parameter and dufour number on the Nusselt number. The Nusselt number increases as the $\mathrm{Q}$ or $\mathrm{Q}_{1}$ or Du increases and decreases as the radiation parameter increases. This shows that the surface heat transfer from the porous plate increases with the increasing values of $Q_{\text {or }} Q_{1}$ or $\mathrm{Du}$ and decreases with increasing values of Nr. Table 3 shows that the effect of increasing the $\mathrm{Sc}$ and $\mathrm{Kr}$ is to increase the rate of mass transfer. These results are in good agreement with Oahimire and Olajuwon [23].

\section{Conclusions}

An analytical study of the MHD heat and mass transfer flow of an incompressible, electrically conducting micropolar fluid with chemical reaction over an infinite vertical porous plate through porous medium in the presence of radiation absorption and Dufour effects is considered. The results are discussed through graphs and tables for different values of parameters entering into the problem. Following conclusions can be drawn from the results obtained:

1. In the presence of a uniform magnetic field, an increase in the strength of the applied magnetic field decelerates the fluid motion along the wall of the plate inside the boundary layer.

2. An increase in Hall current increases the momentum and microrotational velocity.

3. The velocity, microrotational velocity and temperature of the fluid increase with an increase in the radiation absorption parameter, while they decrease with an increase in the heat sink parameters.

4. Both the skin friction and couple stress decrease with an increase in the heat sink parameter or radiation absorption parameter.

5. The Nusselt number increase as the radiation absorption increases and decreases as the radiation parameter increases.

6. The Sherwood number increases as the Schmidt number or chemical reaction parameter increases. 


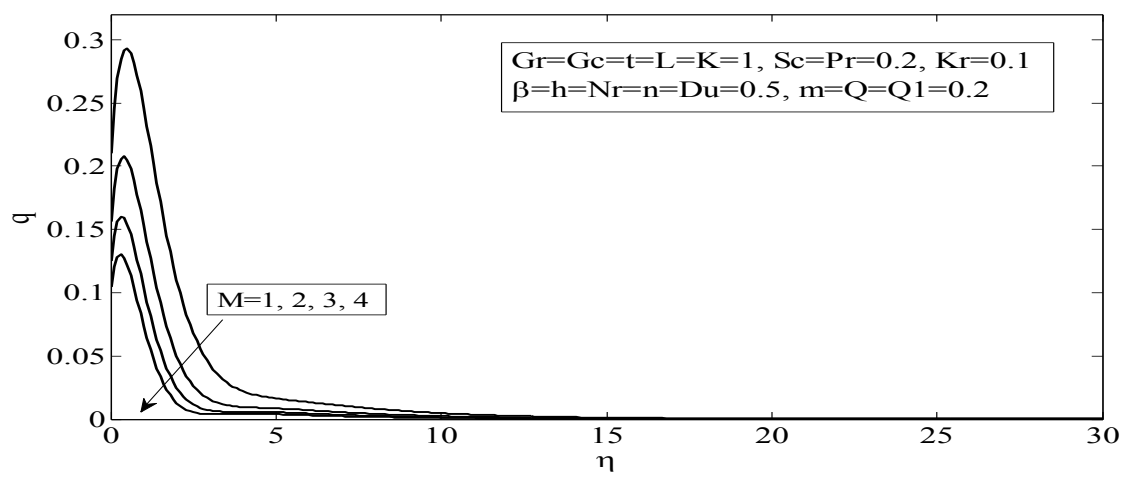

Fig.1 Velocity for different values of $M$

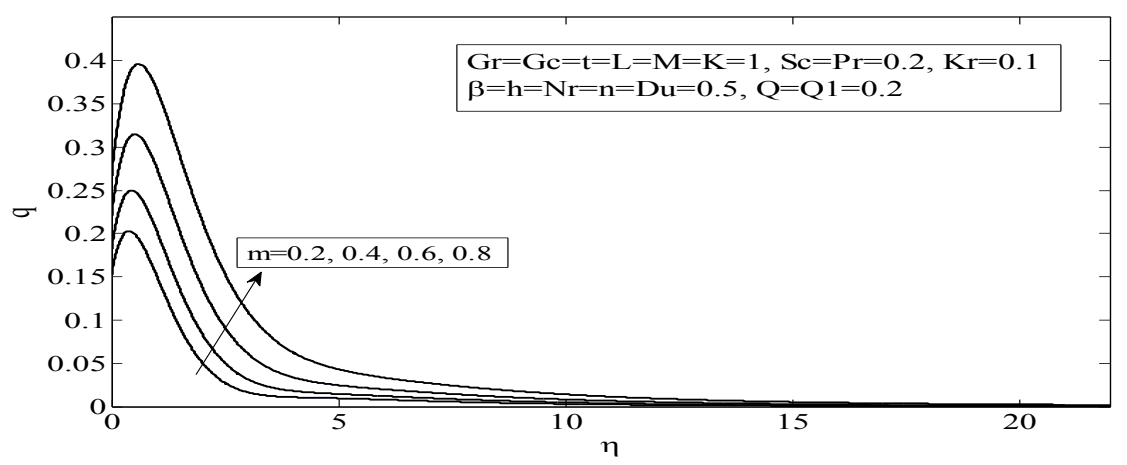

Fig.2 Velocity for different values of $\mathrm{m}$

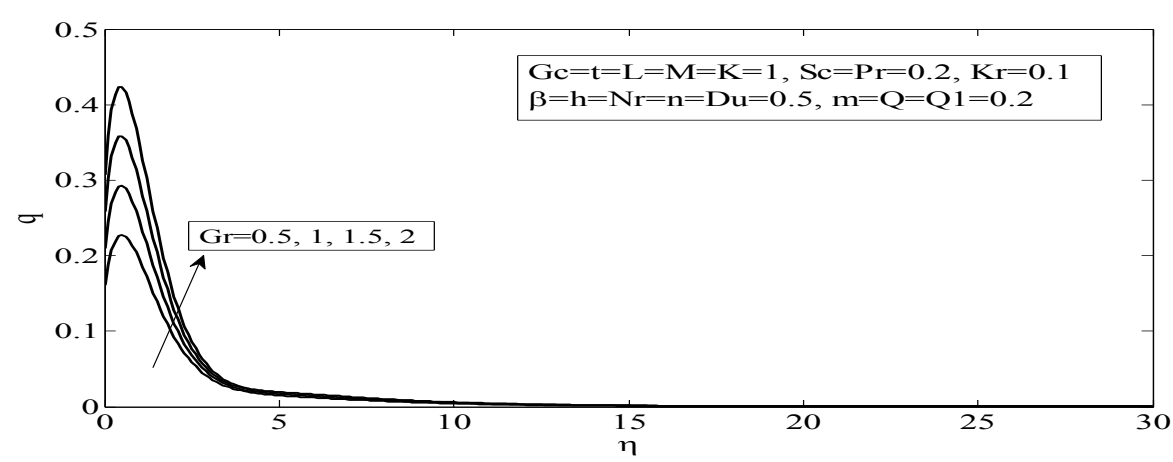

Fig.3 Velocity for different values of $\mathbf{G r}$

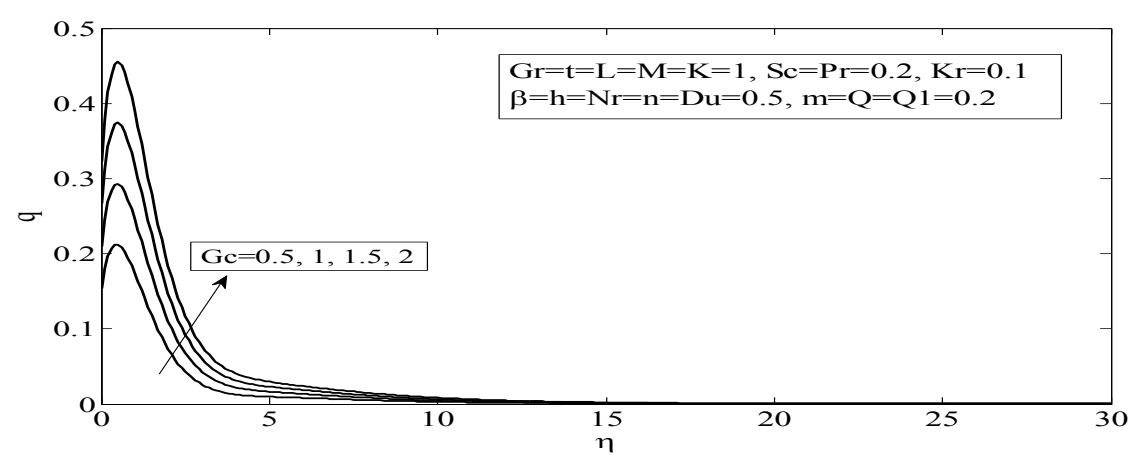

Fig.4 Velocity for different values of Gc 


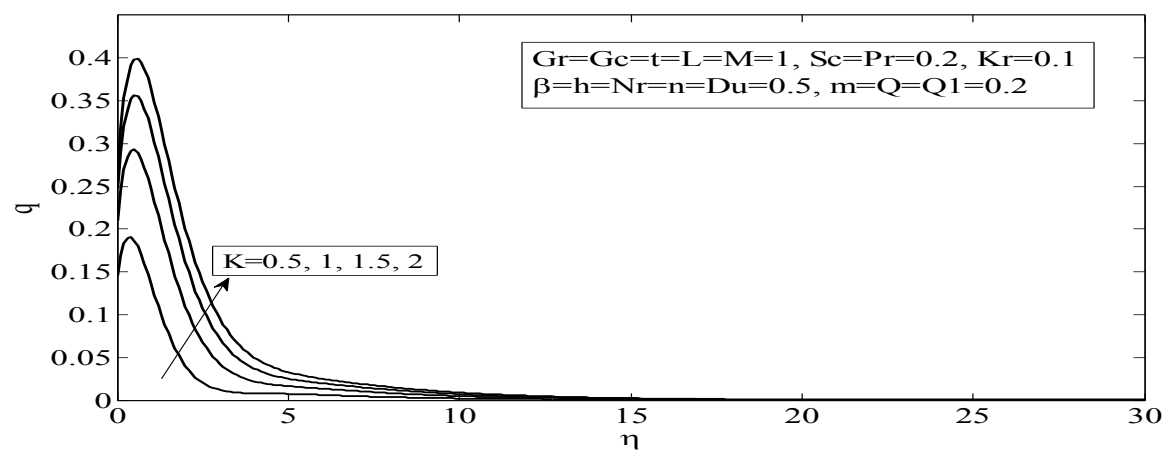

Fig.5 Velocity for different values of $\mathrm{K}$

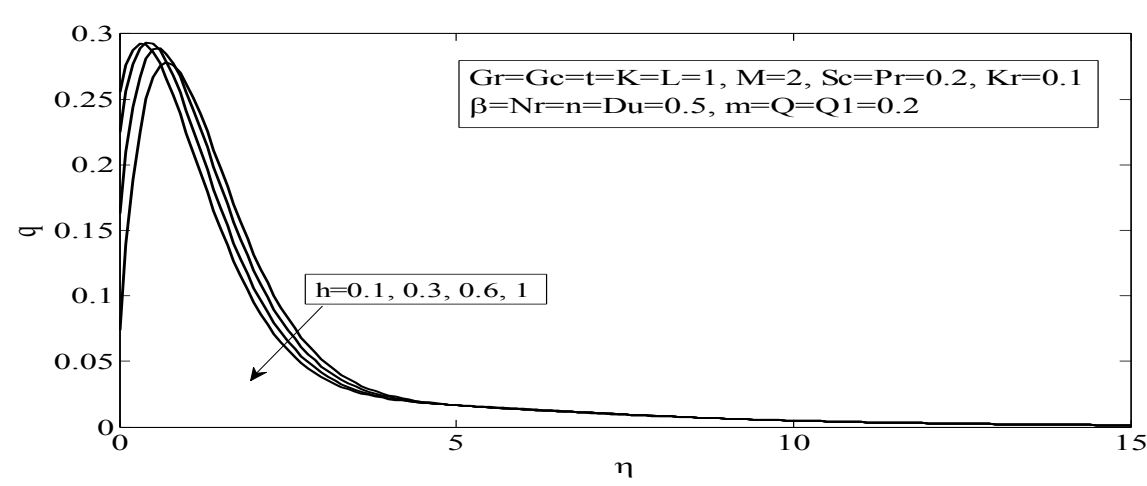

Fig.6 Velocity for different values of $h$

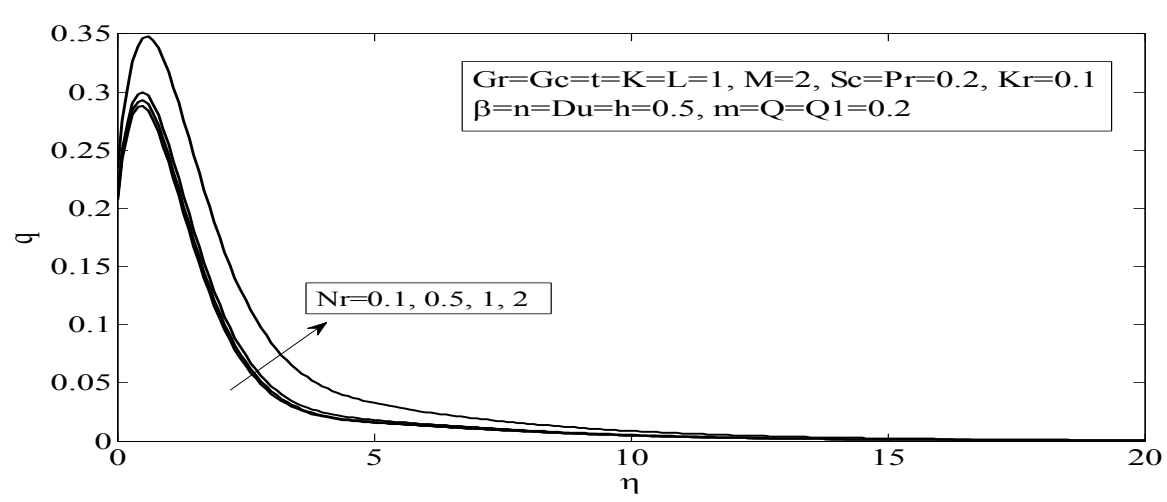

Fig.7 Velocity for different values of $\mathrm{Nr}$

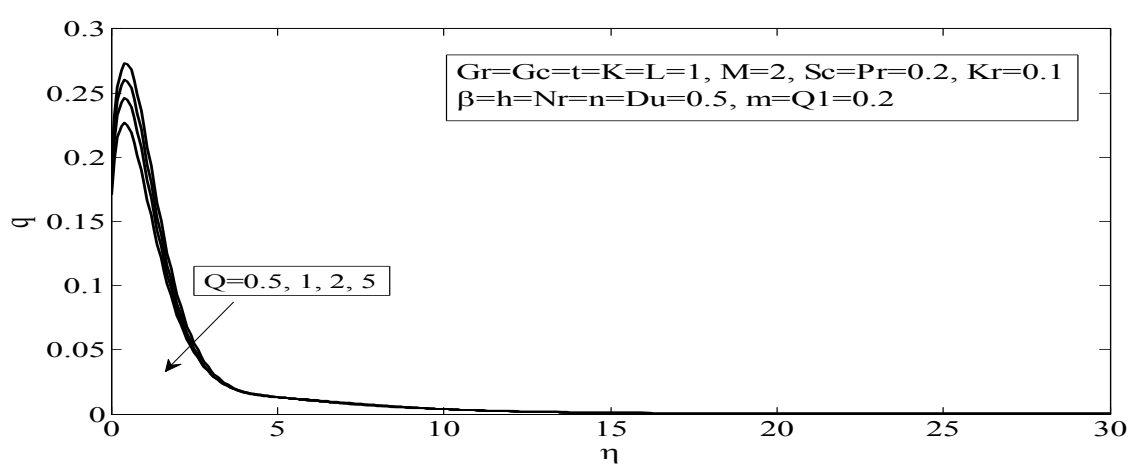

Fig.8 Velocity for different values of $Q$ 


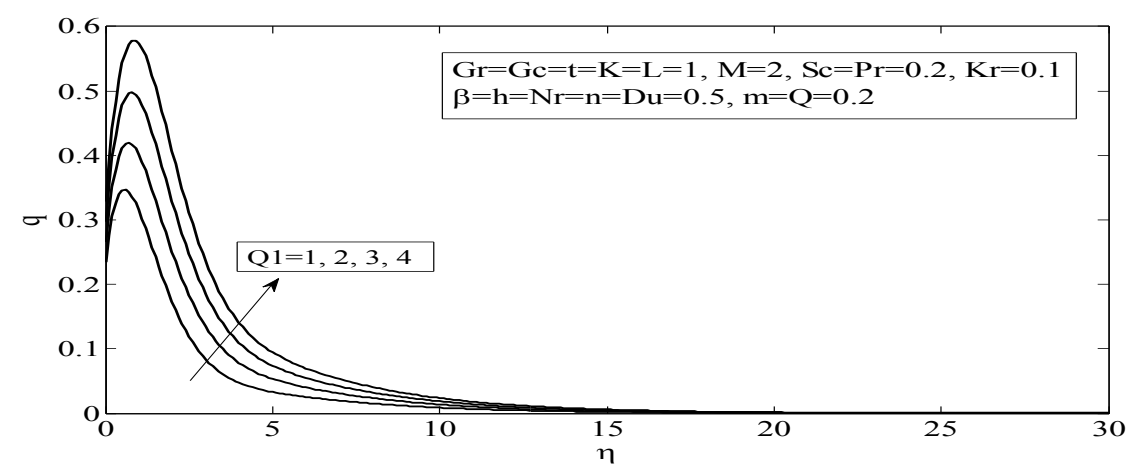

Fig.9 Velocity for different values of $Q_{1}$

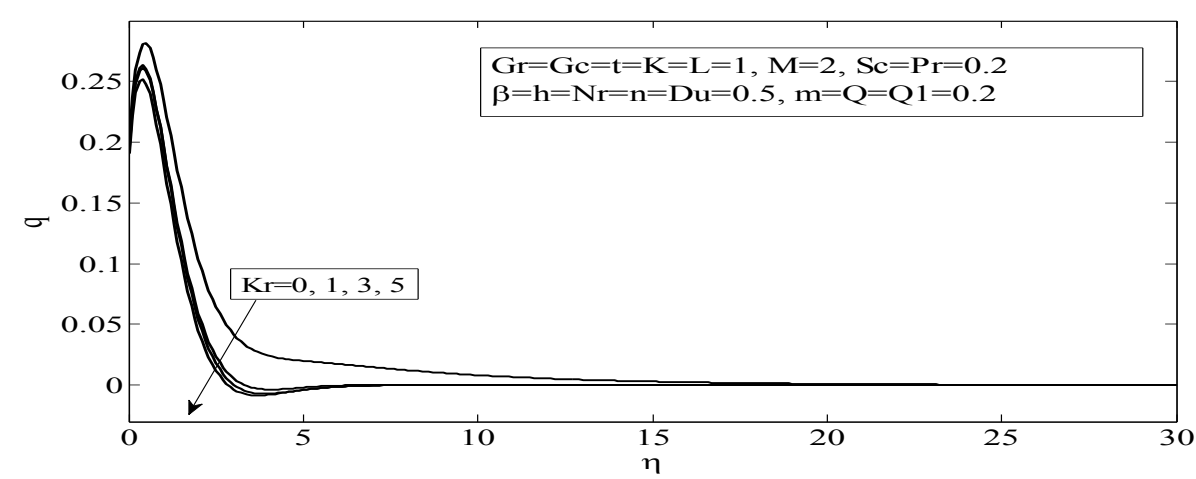

Fig.10 Velocity for different values of $\mathbf{K r}$

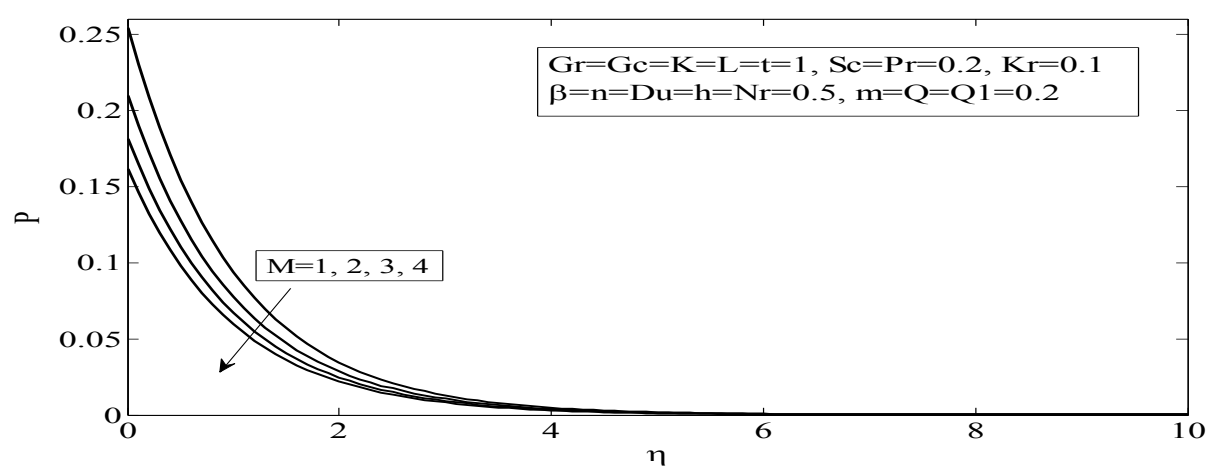

Fig.11(a) Microrotation for different values of $M$ when $n=0.5$

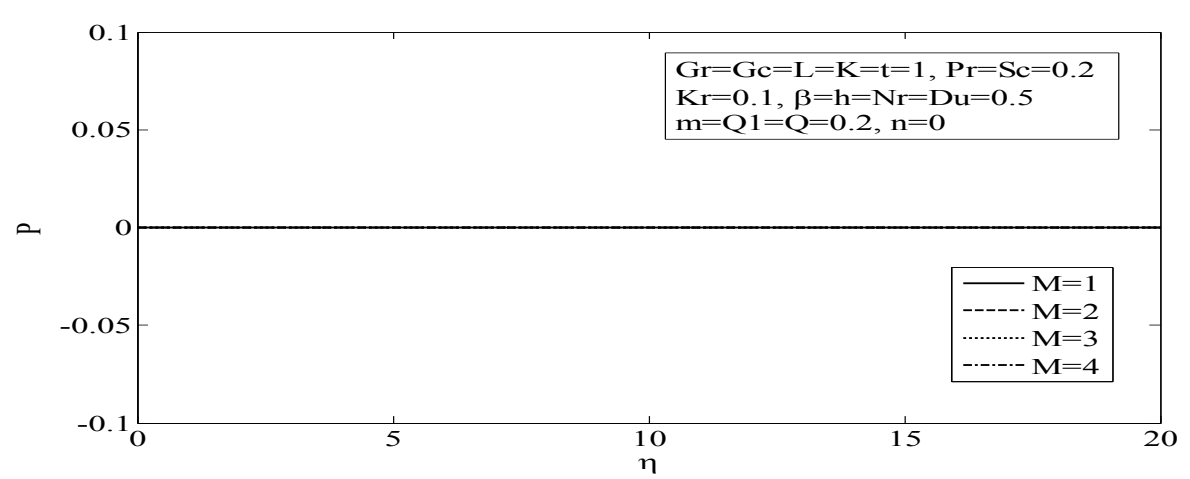

Fig.11(a) Microrotation for different values of $M$ when $n=0$ 


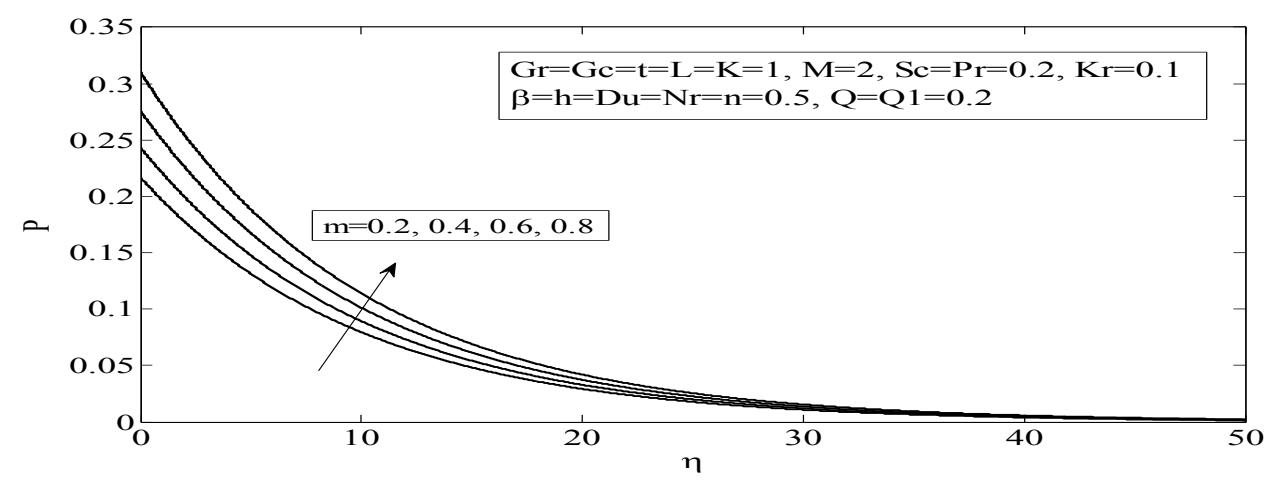

Fig.12 Microrotation for different values of $\mathbf{m}$

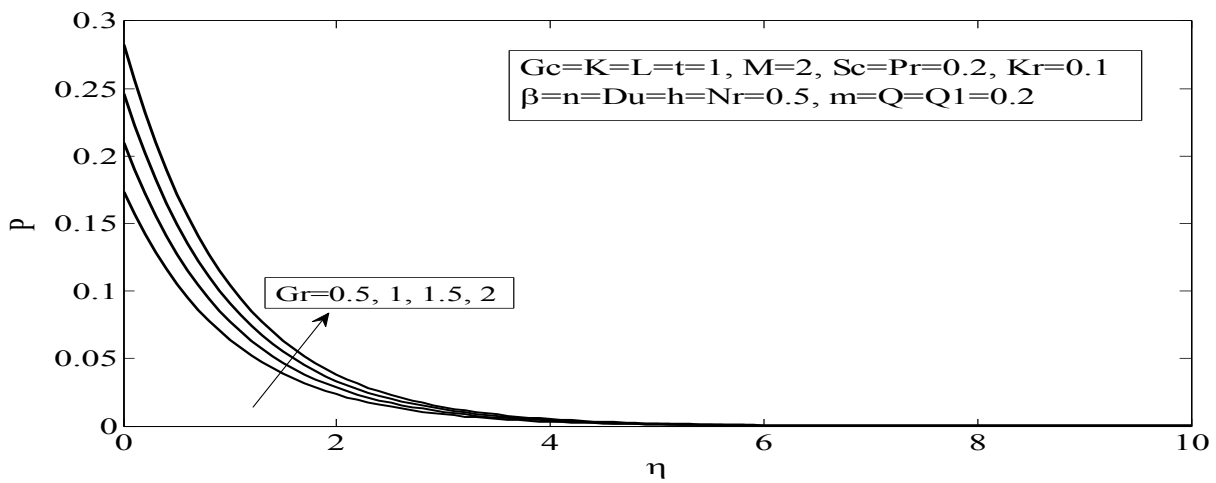

Fig.13 Microrotation for different values of $\mathbf{G r}$

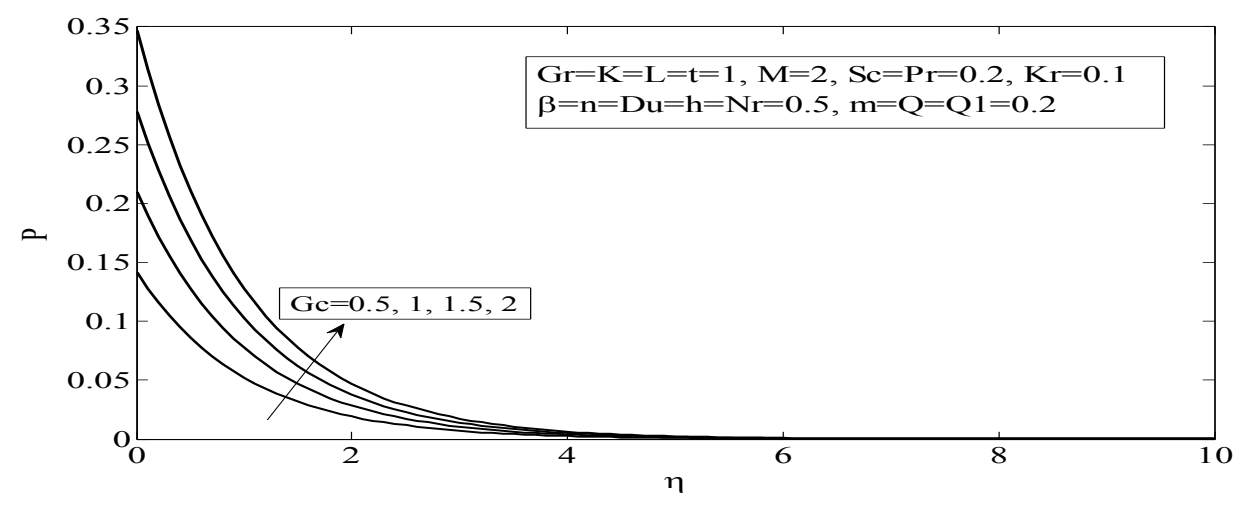

Fig.14 Microrotation for different values of Gc

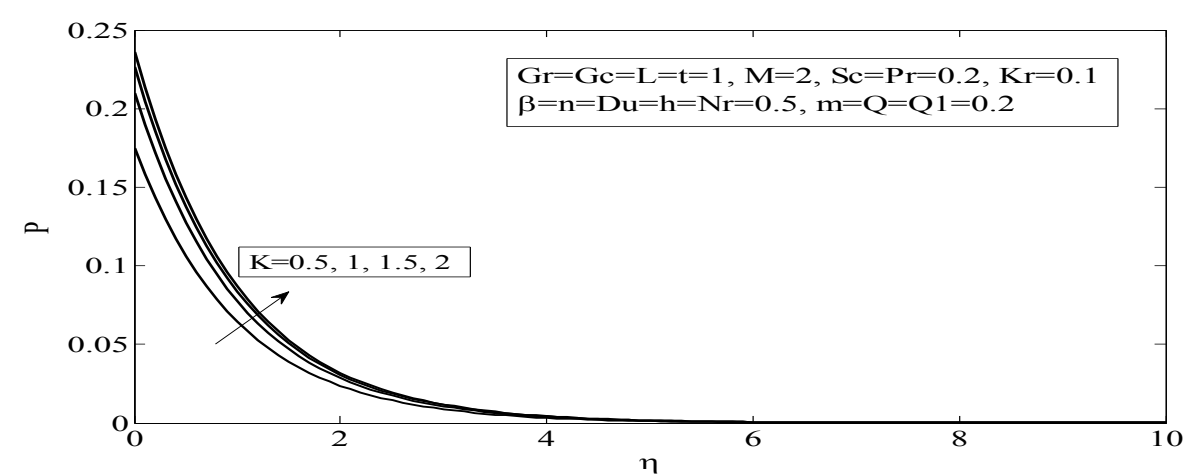

Fig.15 Microrotation for different values of $\mathrm{K}$ 


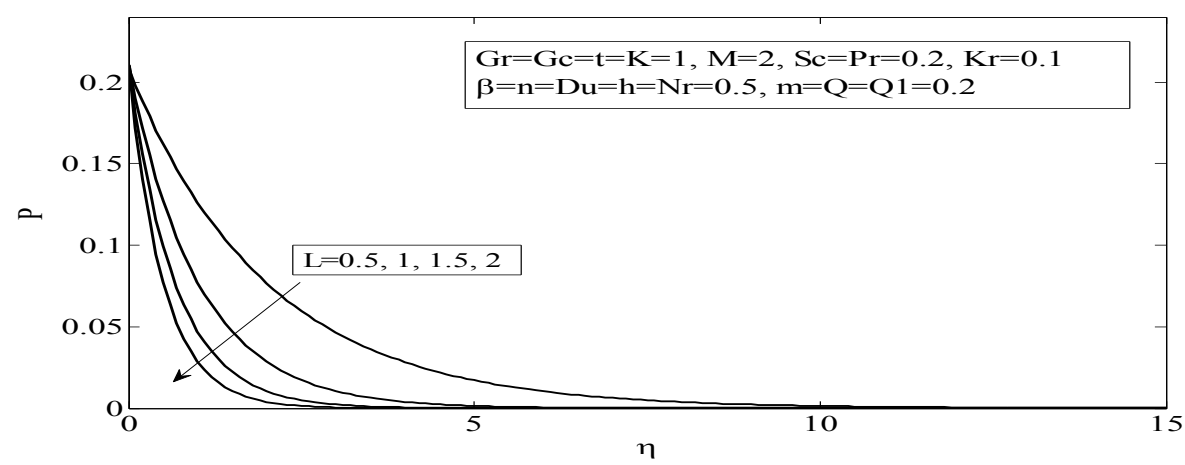

Fig.16 Microrotation for different values of $L$

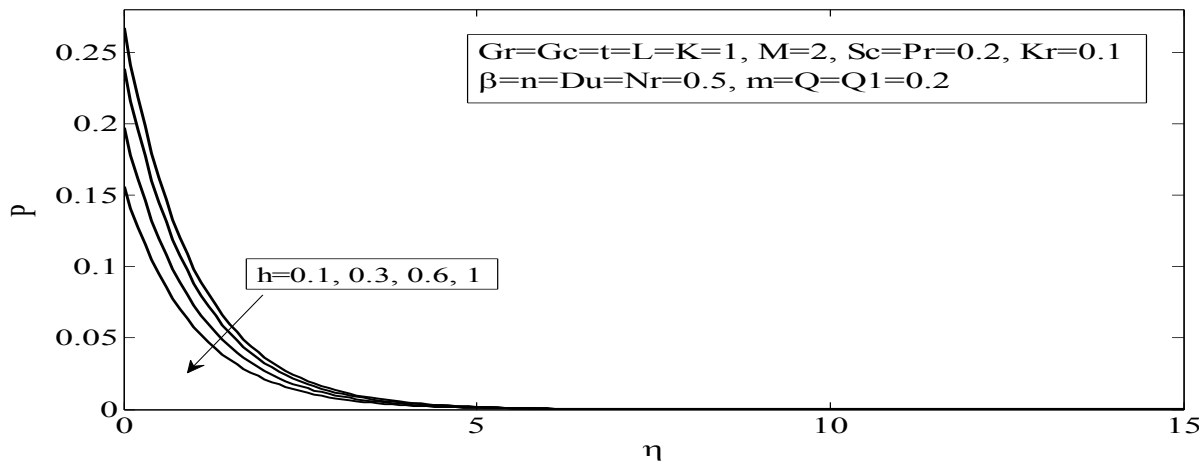

Fig.17 Microrotation for different values of $h$

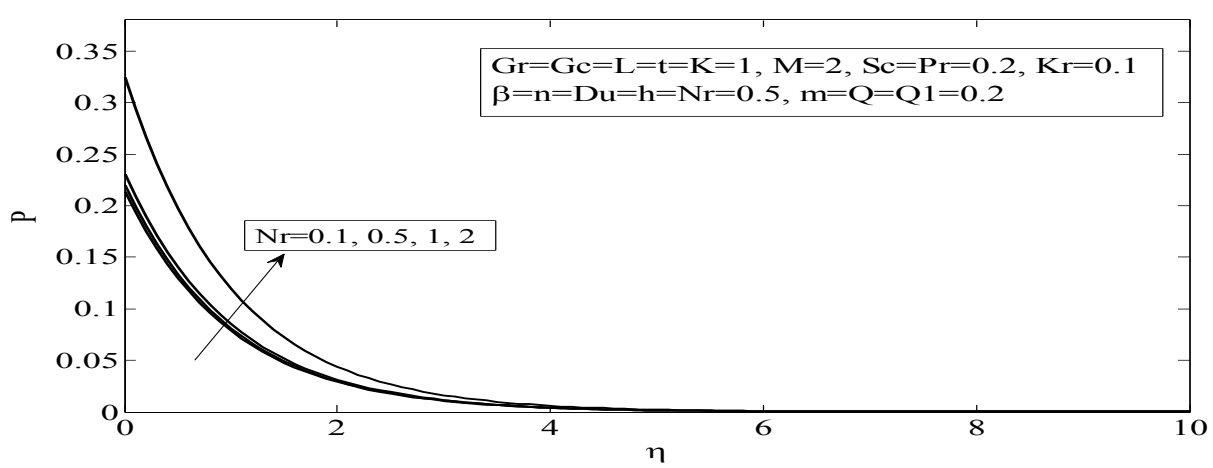

Fig.18 Microrotation for different values of $\mathrm{Nr}$

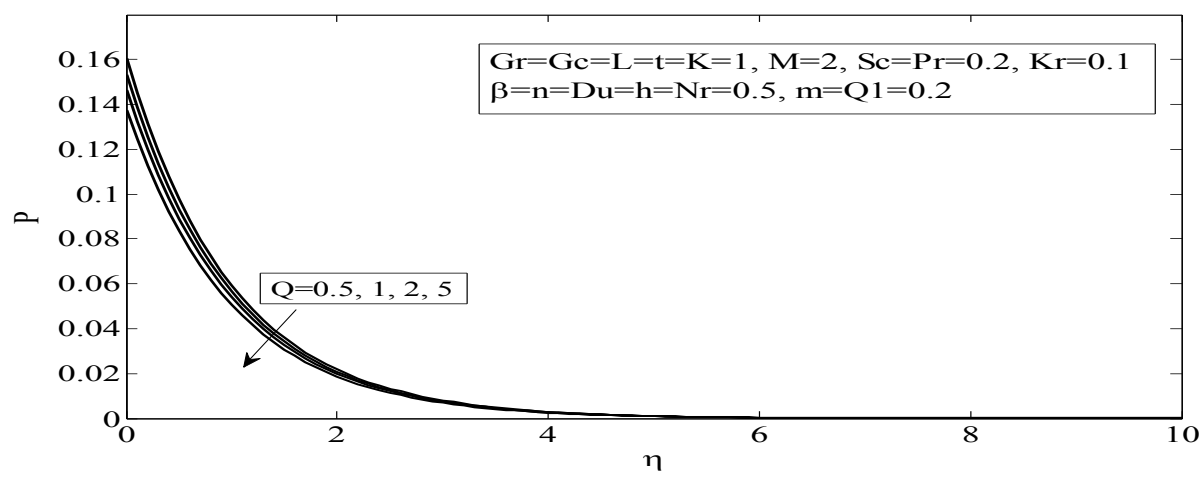

Fig.19 Microrotation for different values of $Q$ 


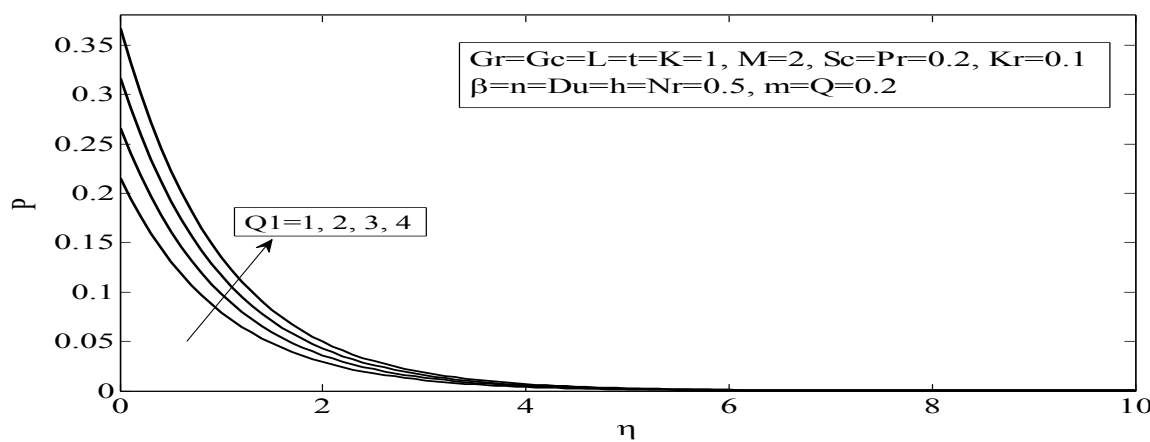

Fig.20 Microrotation for different values of $Q_{1}$

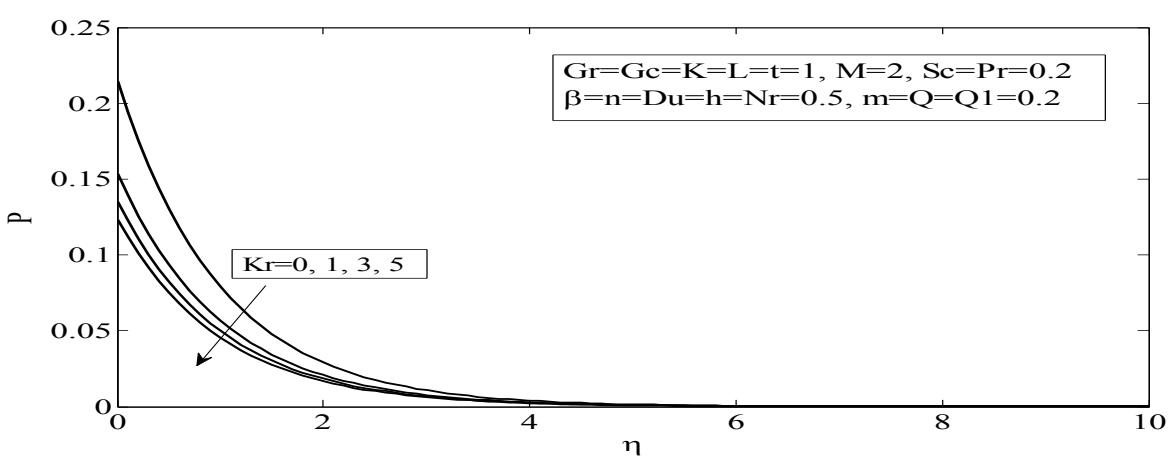

Fig.21 Microrotation for different values of $\mathbf{K r}$

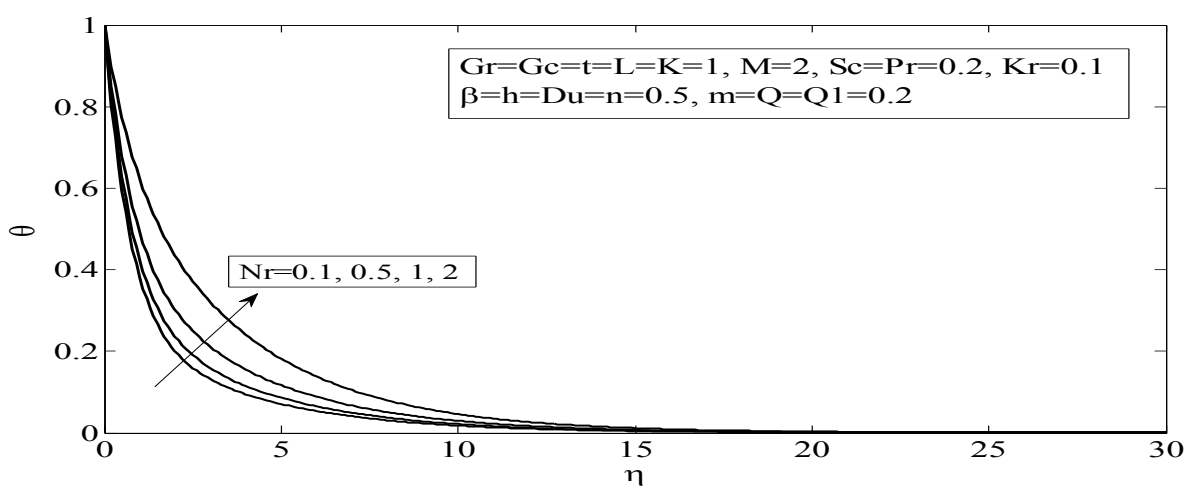

Fig.22 Temperature for different values of $\mathrm{Nr}$

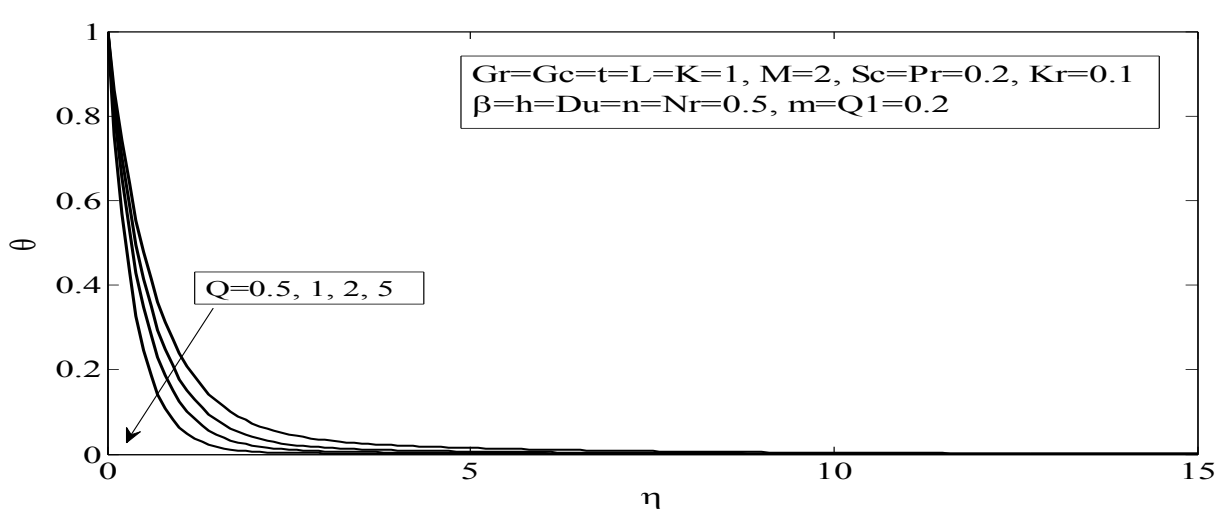

Fig.23 Temperature for different values of $Q$ 


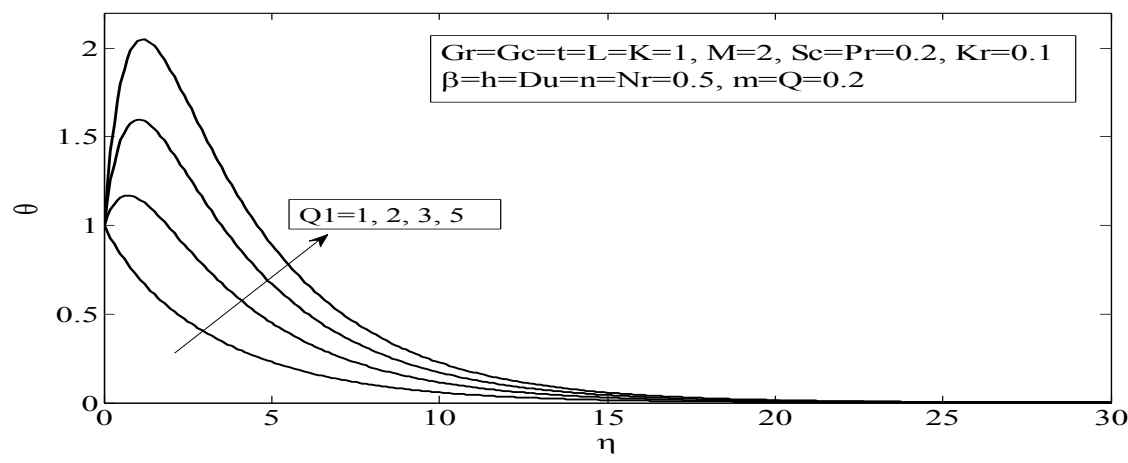

Fig.24 Temperature for different values of $Q_{1}$

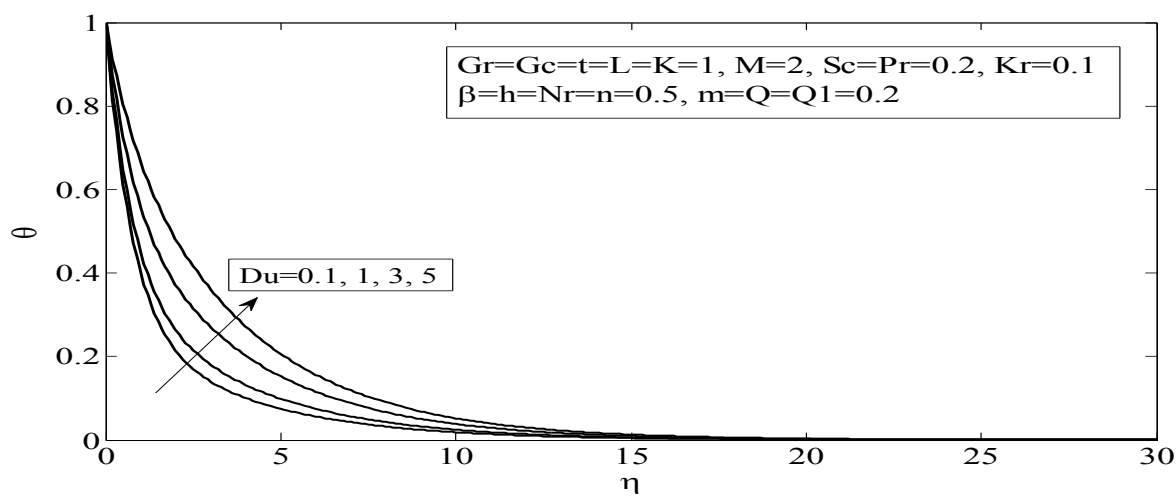

Fig.25 Temperature for different values of Du

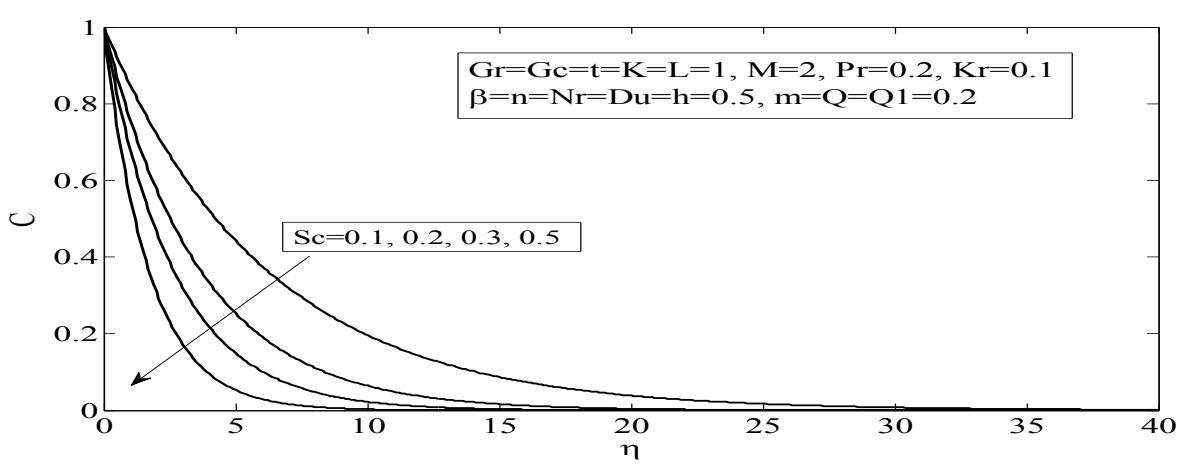

Fig.26 Concentration for different values of Sc

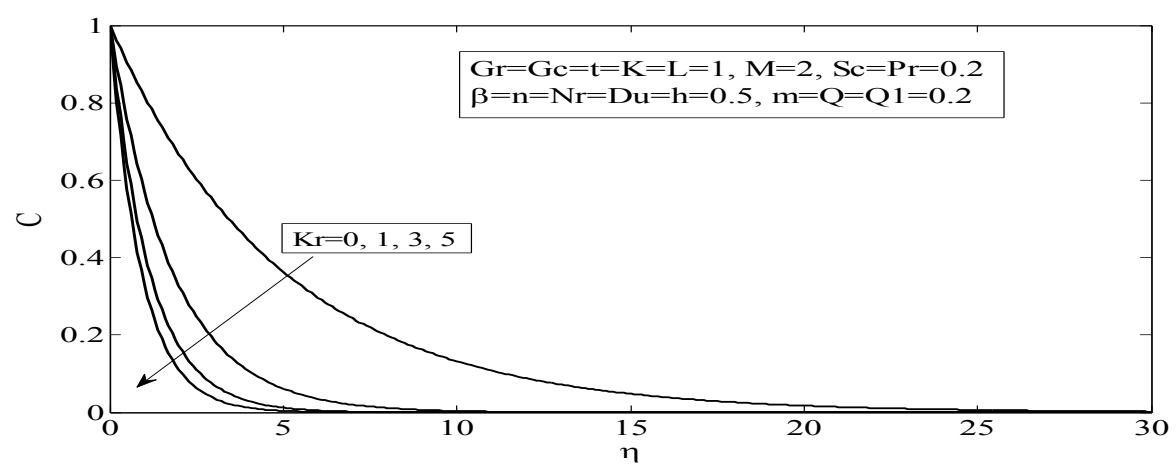

Fig.27 Concentration for different values of $\mathbf{K r}$ 
Table 1 Effect of $\mathrm{n}, \mathrm{M}, \mathrm{Nr}, \mathrm{m}, \mathrm{Q}, \mathrm{Q}_{1}$ and $\mathrm{Kr}$ on $C_{f}$ and $C^{\prime}{ }_{w}$ with $\mathrm{K}=1, \mathrm{t}=1, \omega=0.01, \mathrm{~h}=0.2, \beta=0.5, \mathrm{Du}=0.5$, $\mathrm{Gr}=1, \mathrm{Gc}=1, \operatorname{Pr}=1, \mathrm{Sc}=1, \mathrm{~L}=1$.

\begin{tabular}{|c|c|c|c|c|c|c|c|c|}
\hline $\mathrm{n}$ & $\mathrm{M}$ & $\mathrm{Nr}$ & $\mathrm{m}$ & $\mathrm{Q}$ & $\mathrm{Q}_{1}$ & $\mathrm{Kr}$ & $C_{f}$ & $-C^{\prime}{ }^{\prime}$ \\
\hline 0 & 2 & 0.5 & 0.2 & 0.2 & 0.2 & 0.1 & 0.7594 & 0.0000 \\
0.5 & 2 & 0.5 & 0.2 & 0.2 & 0.2 & 0.1 & 0.5789 & 0.0857 \\
1 & 2 & 0.5 & 0.2 & 0.2 & 0.2 & 0.1 & 0.3966 & 0.1321 \\
\hline 0.5 & 1 & 0.5 & 0.2 & 0.2 & 0.2 & 0.1 & 0.6857 & 0.0633 \\
0.5 & 2 & 0.5 & 0.2 & 0.2 & 0.2 & 0.1 & 0.5789 & 0.0857 \\
0.5 & 3 & 0.5 & 0.2 & 0.2 & 0.2 & 0.1 & 0.5108 & 0.0936 \\
0.5 & 4 & 0.5 & 0.2 & 0.2 & 0.2 & 0.1 & 0.4603 & 0.0959 \\
\hline 0.5 & 2 & 1.0 & 0.2 & 0.2 & 0.2 & 0.1 & 0.6355 & 0.1186 \\
0.5 & 2 & 1.5 & 0.2 & 0.2 & 0.2 & 0.1 & 0.6533 & 0.1292 \\
0.5 & 2 & 2.0 & 0.2 & 0.2 & 0.2 & 0.1 & 0.6623 & 0.1347 \\
\hline 0.5 & 2 & 0.5 & 0.4 & 0.2 & 0.2 & 0.1 & 0.6417 & 0.0739 \\
0.5 & 2 & 0.5 & 0.6 & 0.2 & 0.2 & 0.1 & 0.7231 & 0.0534 \\
0.5 & 2 & 0.5 & 0.8 & 0.2 & 0.2 & 0.1 & 0.8225 & 0.0247 \\
\hline 0.5 & 2 & 0.5 & 0.2 & 0.3 & 0.2 & 0.1 & 0.5934 & 0.0963 \\
0.5 & 2 & 0.5 & 0.2 & 0.4 & 0.2 & 0.1 & 0.5618 & 0.0797 \\
0.5 & 2 & 0.5 & 0.2 & 0.5 & 0.2 & 0.1 & 0.5040 & 0.0494 \\
\hline 0.5 & 2 & 0.5 & 0.2 & 0.2 & 0.3 & 0.1 & 0.6050 & 0.1016 \\
0.5 & 2 & 0.5 & 0.2 & 0.2 & 0.4 & 0.1 & 0.5949 & 0.0955 \\
0.5 & 2 & 0.5 & 0.2 & 0.2 & 0.5 & 0.1 & 0.5848 & 0.0894 \\
\hline 0.5 & 2 & 0.5 & 0.2 & 0.2 & 0.2 & 0.2 & 0.5955 & 0.0953 \\
0.5 & 2 & 0.5 & 0.2 & 0.2 & 0.2 & 0.3 & 0.6025 & 0.0995 \\
0.5 & 2 & 0.5 & 0.2 & 0.2 & 0.2 & 0.4 & 0.6052 & 0.1014 \\
\hline
\end{tabular}

Table 2 Effect of $\mathrm{Nr}, \mathrm{Q}, \mathrm{Q}_{1}$ and $\mathrm{Du}$ on $N u \mathrm{Re}_{x}^{-1}$ with $\mathrm{K}=1, \mathrm{t}=1, \omega=0.01, \mathrm{~m}=0.2, \mathrm{n}=0.5, \mathrm{M}=2, \mathrm{~h}=0.2, \beta=0.5$,

\begin{tabular}{|c|c|c|c|c|}
\multicolumn{6}{|c}{$\mathrm{Gr}=1, \mathrm{Gc}=1, \mathrm{Pr}=1, \mathrm{Kr}=0.1, \mathrm{~L}=1}$. \\
\hline $\mathrm{Nr}$ & $\mathrm{Q}$ & $\mathrm{Q}_{1}$ & $\mathrm{Du}$ & $N u \mathrm{Re}_{x}^{-1}$ \\
\hline 0.5 & 0.2 & 0.2 & 0.5 & 2.1330 \\
1.0 & 0.2 & 0.2 & 0.5 & 1.6277 \\
1.5 & 0.2 & 0.2 & 0.5 & 1.4652 \\
2.0 & 0.2 & 0.2 & 0.5 & 1.3815 \\
\hline 0.5 & 0.3 & 0.2 & 0.5 & 2.4820 \\
0.5 & 0.4 & 0.2 & 0.5 & 2.9972 \\
0.5 & 0.5 & 0.2 & 0.5 & 3.9452 \\
\hline 0.5 & 0.2 & 0.3 & 0.5 & 2.2131 \\
0.5 & 0.2 & 0.4 & 0.5 & 2.2931 \\
0.5 & 0.2 & 0.5 & 0.5 & 2.3731 \\
\hline 0.5 & 0.2 & 0.2 & 0.8 & 2.4202 \\
0.5 & 0.2 & 0.2 & 1.0 & 2.6117 \\
0.5 & 0.2 & 0.2 & 1.2 & 2.8031 \\
\hline
\end{tabular}

Table 3 Comparison of Sherwood number for different Sc and $\mathrm{Kr}$ with $\mathrm{K}=1, \mathrm{t}=1, \omega=0.01, \mathrm{~m}=0.2, \mathrm{n}=0.5$,

\begin{tabular}{|c|c|c|c|}
\hline \multirow[t]{2}{*}{$\mathrm{Sc}$} & \multirow[t]{2}{*}{$\mathrm{Kr}$} & Present study & $\begin{array}{c}\text { Oahimire and } \\
\text { Olajuwon [23] }\end{array}$ \\
\hline & & $\operatorname{Sh} \mathrm{Re}_{x}^{-1}$ & $S h \operatorname{Re}_{x}^{-1}$ \\
\hline 2 & 0.1 & 2.0976 & 2.0953 \\
\hline 3 & 0.1 & 3.0991 & 3.0967 \\
\hline 4 & 0.1 & 4.0998 & 4.0974 \\
\hline 1 & 0.2 & 1.1726 & 1.1707 \\
\hline 1 & 0.3 & 1.2432 & 1.2415 \\
\hline 1 & 0.4 & 1.3077 & 1.3061 \\
\hline
\end{tabular}




\section{References}

[1]. Eringe, A. C., (1964), "Heat Simple micropolar fluids,” International Journal of Engineering Science, Vol. 2, pp. $205-217$.

[2]. Eringen, A. C., (1966), "Theory of micropolar fluids," Vol. 16, pp. 1-18.

[3]. Eringen, A. C., (1972), "Theory of thermomicrofluids," Journal of Mathematical Analysis and Applications, Vol. 38, No. 2, pp. 480-496.

[4]. Ariman, T., Turk, M. A., and Sylvester, N. D., (1973), “Microcontinuum fluid mechanics-a review,” International Journal of Engineering Science, Vol. 11, No. 8, pp. 905-930.

[5]. Ariman, T., Turk, M. A., and Sylvester, N. D., (1974), “Applications of microcontinuum fluid mechanics,” International Journal of Engineering Science, Vol. 12, No. 4, pp. 273-293.

[6]. Lukaszewicz, G., (1999), Micropolar Fluids, Birkh“auser, Boston, Mass, USA, Theory and applications.

[7]. Eringen, A. C., (2001), Microcontinum Field Theories, II. Fluent Media, Springer, NewYork, NY, USA

[8]. Mohamed, I.A., Othman, Zaki S.A., (2008), Thermal instability in a rotating micropolar viscoelastic fluid layer under the effect of electric field, Mech Mech Eng., Vol.12, No.2:171-184.

[9]. Ahmed, N., Kalita, D., (2011), Transient MHD free convection from an infinite vertical porous plate in a rotating system with mass transfer and Hall current, J Energy, Heat Mass Transfer, Vol.33, 271-292.

[10]. Hayat, T., Khan, S.B., Sajid, M., and Asghar, S., (2007), Rotating flow of a third grade fluid in a porous space with Hall current, Nonlinear Dyn., Vol.49, pp.83-91.

[11]. Neela Rani, S.K., Tomar., (2010), Thermal convection problem of micropolar fluid subjected to hall current, Appl Math Model, Vol.34, pp.508-519.

[12]. Eckert, E.R.G., and Drake, R.M., (1972), Analysis of Heat and Mass Transfer, Mc. Graw-.Hill, New York.

[13]. Alam, M.S., and Rahman, M.M., (2005), Dufour and Soret effects on MHD free convective heat and mass transfer flow past a vertical flat plate embedded in porous medium, Journal Naval Architecture and Marine Engineering, Vol. 2(1), pp.55-65.

[14]. Sedeek, M.A., (2004), Thermal-diffusion and diffusion-thermo effects on mixed free- forced convective flow and mass transfer over accelerating surface with a heat source in the presence of suction and blowing in the case of variable viscosity, Acta Mechanica, Vol. 172. pp. 83-94.

[15]. Nazmul, I., and Mahmud, A., (2007), Dufour and Soret effects on steady MHD free convection and mass transfer fluid flow through a porous medium in a rotating system, Journal of Naval Architecture and Marine Engineering, Vol. 4(1), pp. 119.

[16]. Kafoussias, N.G., and Williams, E.M., (1995), Thermal-diffusion and diffusion-thermo effects on mixed free forced convective and mass transfer boundary layer flow with temperature dependent, International Journal of Engineering Science, Vol.33(9), pp. 13691384 .

[17]. Singh, G., Sharma, P.R., and Chamkha, A.J., (2010), "Effect of Volumetric Heat Generation/Absorption on Mixed Convection Stagnation Point Flow on an Isothermal Vertical Plate in Porous Media", Int. J. Industrial Mathematics, Vol.2(2), pp.59-71.

[18]. Das, S.S., Satapathy, A., Das, J.K., and Panda, J.P., (2009), Mass transfer effects on MHD flow and heat transfer past a vertical porous plate through a porous medium under oscillatory suction and heat source, Int. J. Heat Mass Transfer, Vol.52, pp.5962-5969.

[19]. Das S.S., Tripathy, U.K., and Das, J.K., (2010), Hydromagnetic convective flow past a vertical porous plate through a porous medium with suction and heat source, International Journal of Energy and Environment, Vol.1(3), pp.467-478.

[20]. Khan, K., (2006), Heat transfer in a viscoelastic fluid flow over a stretching surface with heat source/sink, suction/blowing and radiation, Int. J. Heat Mass Trans., Vol.49, pp.628-639.

[21]. Pal and Talukdar. B., (2010), Perturbation analysis of unsteady magnetohydrodynamic convective heat and mass transfer in a boundary layer slip flow past a vertical permeable plate with thermal radiation and chemical reaction, Commun. Nonlin. Sci. Numer. Simul., Vol.15, pp.1813-1830.

[22]. Elgazery, N. S., (2009), "The effects of chemical reaction, hall and ion-slip currents on MHD flow with temperature dependent viscosity and thermal diffusivity," Communications in Nonlinear Science and Numerical Simulation, Vol. 14, No. 4, pp. 1267-1283.

[23]. Oahimire, J.I., Olajuwon, B.I., (2013), Effect of Hall current and thermal radiation on heat and mass transfer of a chemically reacting MHD flow of a micropolar fluid through a porous medium, A Journal of King Saud University - Engineering Sciences.

[24]. Satya Narayana, P.V., Venkateswarlu, B., Venkataramana, S., (2013), Effects of Hall current and radiation absorption on MHD micropolar fluid in a rotating system, Ain Shams Engineering Journal, Vol.4, pp.843-854.

[25]. Motsa, S. S., and Shateyi, S., (2012), The Effects of Chemical Reaction, Hall, and Ion-Slip Currents on MHD Micropolar Fluid Flow with Thermal Diffusivity Using a Novel Numerical Technique, Hindawi Publishing Corporation Journal of Applied Mathematics, Volume 2012, Article ID 689015, 30 pages.

[26]. Salem, A. M., (2013), The Effects of Variable Viscosity, Viscous Dissipation and Chemical Reaction on Heat and Mass Transfer Flow of MHD Micropolar Fluid along a Permeable Stretching Sheet in a Non-Darcian Porous Medium, Hindawi Publishing Corporation Mathematical Problems in Engineering, Volume 2013, Article ID 185074, 10 pages 